\title{
Does a patient with a thunderclap headache need a lumbar puncture?
}

\author{
Ben Fine MD MSc, Navneet Singh MD, Richard Aviv MBChB, R. Loch Macdonald MD PhD
}

\begin{abstract}
A 37-year-old woman presents to the emergency department four hours after an acute onset of the "worst headache of her life" that began at rest. She experienced no loss of consciousness or neck pain. She has no history of intracranial disease or family history of cerebral aneurysms. Her vital signs are normal. No evidence of neurologic deficits is found on examination. Subarachnoid hemorrhage is on your differential diagnosis in this neurologically intact patient. You consider the relevant work-up.
\end{abstract}

\section{Can history and physical examination rule out nontraumatic subarachnoid hemorrhage?}

About 1 in 14 patients presenting to the emergency department with a severe acute headache will have a subarachnoid hemorrhage from an aneurysm. ${ }^{1}$ Clinicians are faced with a wide spectrum of clinical presentations in patients with subarachnoid hemorrhage ${ }^{2}$ and have little evidence to help them determine which patients can safely avoid imaging or lumbar puncture.

In an effort to elucidate decision-making in subarachnoid hemorrhage, one large multicentre Canadian trial prospectively studied nearly 2000 patients who presented to an emergency department with a new severe headache that was rapidly peaking (within one hour after onset) or was accompanied by syncope. ${ }^{1}$ The patients had no neurologic deficits. They were followed for six months. The authors' main result - a set of high-risk clinical characteristics that excluded $100 \%$ of subarachnoid hemorrhages $(95 \%$ confidence interval [CI] 97\%-100\%) - awaits external validation.

Meanwhile, when assessing patients with severe acute headache, clinicians can consider evidence from the prospective study to guide clinical decisions. Table 1 lists clinical features whose presence or absence can help in the evaluation for subarachnoid hemorrhage in such patients.

\section{Can computed tomography rule out subarachnoid hemorrhage?}

Since 1998, computed tomography (CT) scanners with multiple axial detectors, which reduce artifacts and improve image resolution, have become standard. ${ }^{2}$ Studies over the past decade have failed to prove that imaging with these multidetector CT scanners can rule out subarachnoid hemorrhage in all instances. However, evidence from a prospective study using multidetector scanners with at least four detectors suggests that negative scans can reliably exclude subarachnoid hemorrhage if obtained within the first six hours after onset of the headache. ${ }^{3}$

The study examined 3123 adults across Canada who had a headache of acute onset (peak within one hour after onset), no neurologic impairment and no known intracranial pathology. ${ }^{3}$ The results showed that CT imaging detected all 121 patients with subarachnoid hemorrhage in the subgroup of 953 who underwent imaging within six hours after onset of the headache (sensitivity $100 \%, 95 \%$ CI 97\%-100\%). All images were interpreted by a neuroradiologist or a general radiologist who routinely reported on head CT images.

Table 1: Clinical features to help determine the likelihood of subarachnoid hemorrhage in neurologically intact patients who present with a severe acute headache that is rapidly peaking or accompanied by syncope*

\begin{tabular}{|lc|}
\hline $\begin{array}{l}\text { Presence of variable increases likelihood } \\
\text { of subarachnoid hemorrhage }\end{array}$ & $\begin{array}{c}\text { Positive } \\
\text { likelihood ratio }\end{array}$ \\
\hline Neck stiffness (flexion/extension) on examination & 5.94 \\
\hline Loss of consciousness, witnessed & 4.59 \\
\hline Loss of consciousness, not witnessed & 3.77 \\
\hline $\begin{array}{l}\text { Absence of variable decreases likelihood } \\
\text { of subarachnoid hemorrhage }\end{array}$ & $\begin{array}{c}\text { Negative } \\
\text { likelihood ratio }\end{array}$ \\
\hline Worst headache of life & 0.31 \\
\hline Complaint of neck stiffness or pain & 0.42 \\
\hline Arrival by ambulance & 0.52 \\
\hline $\begin{array}{l}\text { *Ratios were derived from a study by Perry et al.' This prospective study excluded patients } \\
\text { whose headache began more than 14 days before presentation, as well as patients with } \\
\text { recurrent headaches ( }>\text { three), focal neurologic deficits, papilledema, history of subarachnoid } \\
\text { hemorrhage, aneurysm, hydrocephalus or brain neoplasm. }\end{array}$ \\
\hline
\end{tabular}


Because hemorrhage clears from the cerebrospinal fluid over time, the sensitivity (and consequently also the negative predictive value) of CT imaging decreases over time as well. One study, which included results from some studies using older, single-slice CT scanners, calculated the negative likelihood ratio to be 0.02 if scanning were performed within 12 hours after onset of headache, 0.07 if performed within 24 hours and 0.18 if performed more than 24 hours after onset. ${ }^{4}$ Thus, a patient with a pretest probability of subarachnoid hemorrhage of 1 in 14 would have a probability of about 1 in 700 with a negative CT result if imaging were done within 12 hours after onset of headache, and a probability of about 1 in 80 with a negative result if imaging were performed more than 24 hours after onset.

\section{Can a combination of $\mathrm{CT}$ imaging and lumbar puncture rule out subarachnoid hemorrhage?}

Negative results of CT imaging and lumbar puncture, in combination, can rule out subarachnoid hemorrhage in neurologically intact patients with acute headache. A prospective Canadian study involving 592 patients found no missed cases using a combined approach. ${ }^{5}$ If the result of lumbar puncture is positive (i.e., red blood cells on analysis of the cerebrospinal fluid), even from a "traumatic tap," or if clinicians maintain suspicion of subarachnoid hemorrhage (e.g., recurrent presentations, strong family history or prior history of aneurysm), the patient should undergo CT- or catheter-directed angiography.

\section{Case revisited}

Investigating patients for subarachnoid hemorrhage if they present with an acute, rapidly peaking headache and no neurologic deficits presents a challenge to physicians in the emergency department. In the case we have described, current evidence can help guide appropriate investigation.

The pretest probability of subarachnoid hemorrhage in our patient, who underwent CT scanning within six hours after the onset of her headache, is about $12 \%$ (approximately one in eight). ${ }^{3}$ The absence of neck pain and her arrival by car and not ambulance reduce the likelihood of her having a hemorrhage (Table 1) but do not exclude the possibility. Proposed clinical decision rules ${ }^{1}$ are currently undergoing validation.
Evidence suggests that $\mathrm{CT}$ scans interpreted by a qualified radiologist can exclude subarachnoid hemorrhage if imaging is performed within six hours after onset of headache in select patients, as in our case. Once current evidence is validated, clinicians may reconsider the current practice of performing a lumbar puncture on every patient who has a negative CT result within six hours after presentation.

\section{References}

1. Perry JJ, Stiell IG, Sivilotti ML, et al. High risk clinical characteristics for subarachnoid haemorrhage in patients with acute headache: prospective cohort study. BMJ 2010;341:c5204.

2. Edlow JA, Malek AM, Ogilvy CS. Aneurysmal subarachnoid hemorrhage: update for emergency physicians. J Emerg Med 2008;34:237-51

3. Perry JJ, Stiell IG, Sivilotti ML, et al. Sensitivity of computed tomography performed within six hours of onset of headache for diagnosis of subarachnoid haemorrhage: prospective cohort study. BMJ 2011;343:d4277.

4. Coats TJ, Loffhagen R. Diagnosis of subarachnoid haemorrhage following a negative computed tomography for acute headache: a Bayesian analysis. Eur J Emerg Med 2006;13:80-3.

5. Perry JJ, Spacek A, Forbes M, et al. Is the combination of negative computed tomography result and negative lumbar puncture result sufficient to rule out subarachnoid hemorrhage? Ann Emerg Med 2008;51:707-13.

Affiliations: From the Diagnostic Radiology Program (Fine) and the Diagnostic Radiology Clinician Investigator Program (Singh), Faculty of Medicine, University of Toronto, Toronto, Ont.; the Department of Medical Imaging (Aviv), Sunnybrook Health Sciences Centre and University of Toronto, Toronto, Ont.; and the Division of Neurosurgery (Macdonald), St. Michael's Hospital and University of Toronto, Toronto, Ont.

Contributors: Ben Fine, Navneet Singh and R. Loch Macdonald conceived the paper. All of the authors contributed to the literature search. Ben Fine and Navneet Singh drafted the manuscript and its revisions. All of the authors critically reviewed the paper and approved the final version submitted for publication.

Decisions is a series that focuses on practical evidence-based approaches to common presentations in primary care. The articles address key decisions that a clinician may encounter during initial assessment. The information presented can usually be covered in a typical primary care appointment. Articles should be no longer than 650 words, should include one box, figure or table and should begin with a very brief description (75 words or less) of the clinical situation. The decisions addressed should be presented in the form of questions. A box providing helpful resources for the patient or physician is encouraged. 